\title{
Introduction to the Special Issue, Pathways Between Genes, Brain, and Behavior
}

\author{
William S. Kremen • Kristen C. Jacobson
}

Published online: 13 February 2010

(c) The Author(s) 2010. This article is published with open access at Springerlink.com

\begin{abstract}
In the past 10 years or so, with the sequencing of the human genome and rapid advances in the development of high throughput techniques, the field of behavior genetics has increasingly moved toward the detection of actual genes and environmental factors. However, the field is still in the relatively early stages of understanding some of the basic facts about the complex genetic underpinnings of brain structure and function and their relationship to behavior. The 15 articles in this special issue were selected to represent the diversity of methodologies applied to the complexity of pathways linking genes, brain, and behavior. While providing strong evidence for the role of genes in individual differences in brain structure and function, these papers also demonstrate that environmental experiences alter neurobiological pathways, and that genetic factors may further moderate the impact of environmental experience. Most importantly, the breadth of studies proves that in order to be able to trace the pathways between genes, brain, and behavior, we need experts in genetics, neuroscience, psychology, and psychiatry.
\end{abstract}

Keywords Genes $\cdot$ Brain $\cdot$ Behavior

In the past 10 years or so, with the sequencing of the human genome and rapid advances in the development of

W. S. Kremen

Department of Psychiatry, University of California,

San Diego, La Jolla, CA, USA

K. C. Jacobson $(\square)$

Department of Psychiatry \& Behavioral Neuroscience,

University of Chicago, MC 3077, 5841 S Maryland Ave,

Chicago, IL 60637, USA

e-mail: kjacobso@bsd.uchicago.edu high throughput techniques, the field of behavior genetics has increasingly moved toward the detection of actual genes and environmental factors. However, the field is still in the relatively early stages of understanding some of the basic facts about the complex genetic underpinnings of brain structure and function and their relationship to behavior. Many of the 15 articles in this special issue make it clear that there is still much to be learned about the heritability of basic brain phenotypes and genetic covariation of brain structure and brain activity. Genetic methodologies are quite diverse across studies, and include traditional twin studies of heritability, association analysis, analysis of telomere length, copy number variation (CNV), and the effects of early environmental experiences on the neurobiology of disease and behavior.

Our attempts to better understand these complex pathways linking genes, brain, and behavior necessitate human epidemiological research (Anokhin et al., Bjetmann et al., Gilmore et al., Mechelli et al., Penke et al., Schmitt et al., Smit et al., Wallace et al., Zhang et al.), and human and animal experimental research (Hamidovic et al., Curley et al., Velez et al., Meagher et al., Roubertoux et al.). Also important to advancing our knowledge is the development of novel statistical methodologies that can be used in the analysis of the dynamic, interactive influences that characterize relationships between genes, brain, and behavior (Stoltenberg et al.).

There are several neuroimaging papers using a variety of different techniques. The structural magnetic resonance imaging (MRI) studies all focus on aspects of genetic covariation. Taking a step beyond existing studies of global brain structure measures, Schmitt et al. examined genetic correlations across different brain regions in child and adolescent twins and delineated lobar latent factors. Wallace et al. and Betjemann et al. examined the genetic 
correlations between cognitive abilities and specific brain regions. In a non-twin study, Penke et al. used diffusion tensor imaging to demonstrate a relationship between brain microstructure and cognitive ability. They also found that the ancestral allele ADRB2 SNP (single nucleotide polymorphism) was associated with both white matter integrity in the splenium and with cognitive ability.

Using functional MRI, Mechelli et al. examined brain activation during a perceptual matching task in healthy children in relation to NRG1 genotype. NRG1 is thought to be a susceptibility gene for schizophrenia and bipolar disorder, but it is a question as to how it may affect cortical function in healthy individuals. Although there were no performance differences, these investigators found that having two high-risk alleles did increase activation in a distributed neural network.

Three papers examined brain function by means of electroencephalography (EEG). Smit et al. used EEG to examine the genetic architecture of functional brain connectivity in different age groups. Connectivity indices had moderate to high heritabilities. Connectivity was also more random in adolescents and old age and more structured in middle age, a finding that highlights the importance of age and developmental factors in genetic studies of the brain. Anokhin et al. studied event-related potentials (ERPs) evoked by facial expression in adolescent twins. This is the first study to provide evidence of the heritability of brain activity in response to emotion processing. The results suggest that these ERP measures could serve as endophenotypes for disorders of social cognition and behavior. Gilmore et al. also used EEG/ERPs as potential endophenotypes for externalizing spectrum disorders. Their multivariate twin analyses suggested that there is significant genetic variance common across candidate endophenotypes which is shared, at least in part, with the externalizing spectrum, but also demonstrated significant genetic effects on externalizing that are unrelated to these endophenotypes, as well as some sex-specific genetic effects.

Aggression, a specific form of externalizing behavior, was the subject of two animal studies. Velez et al. compared two substrains of mice (BALB/cJ and BALB/cByJ) and found substantial differences in aggression, but not in other behaviors. Previously reported SNP differences were not replicated, but copy number variants did appear to differentiate aggressive behavior between these two substrains. Roubertoux et al. conducted four experiments aimed at understanding the role of steroid sulfatase (STS) and the steroid sulfatase gene (Sts) in attack behavior. STS concentrations in the liver have been associated with attack behavior. STS also induces low concentrations of sulfated steroids, but paradoxically, injection of a sulfated steroid increases attack behavior. Their studies of behavioral data and transcription activity in liver and brain provide a possible resolution to this paradox.

Two papers used animal studies to investigate the effects of early environmental experiences on the neurobiology of disease and behavior. For example, Meagher et al. showed that early adversity (maternal separation) affected vulnerability to CNS infection later in life, while a separate series of experiments also showed differences in both cortisol and behavioral responses. This series of experiments demonstrates that early stress has the power to alter behavioral, endocrine, and immune response to infection. In a related vein, Curley et al. conducted a series of experiments involving manipulations of both post-natal and postweaning environments in different genetic strains of mice and found strain $\mathrm{x}$ environment interactions in adult differences in anxiety-like behavior. This paper also showed that maternal behavior is dependent on pup strain. As a whole, the experiments in this paper validate the importance of gene $\times$ environment interaction in the development of individual differences in behavior. Moreover, the long-term goals of these research programs are to identify the mechanisms through which early environmental experiences can alter vulnerabilities to behavioral and infectious disorders, and to identify how genes may further moderate these pathways.

The breadth of methodologies is also reflected in a paper using actual brain tissue and one based on a human experimental design. In Zhang et al., variations in telomere length were examined in cerebellar grey matter among groups of schizophrenia, bipolar, and depressed patients, and healthy controls. In contrast to prior research using telomere length in lymphocytes, no significant group differences emerged. In Hamidovic et al., healthy patients were given varying doses of amphetamines, and genetic differences in drug response were examined. Polymorphisms in a dopamine transmitter SNP were related to individual differences in stimulation and feelings of euphoria. In our final paper, Stoltenberg and Nag present the description and evidence for validation of a method for examining genetic variation in the serotonin system that acknowledges the dynamic and multi-level nature of neurotransmitter systems.

This special issue highlights the complex nature of the pathways between genes, brain, and behavior. While providing strong evidence for the role of genes in individual differences in brain structure and function, these papers also demonstrate that environmental experiences alter neurobiological pathways, and that genetic factors may further moderate the impact of environmental experience. Most importantly, the breadth of studies proves that in order to be able to trace the pathways between genes, brain, and behavior, we need experts in genetics, neuroscience, psychology, and psychiatry. We hope that this special 
issue, with its diverse group of researchers and methodologies, is a step towards the collaborations needed to expand our understanding of the underpinnings of individual differences in the neurobiology of behavior and disease.
Open Access This article is distributed under the terms of the Creative Commons Attribution Noncommercial License which permits any noncommercial use, distribution, and reproduction in any medium, provided the original author(s) and source are credited. 